\title{
ISOTOPIE DELLA NAZIONE E DELLA PATRIA LOCALE IN LUIGI MENEGHELLO
}

\section{FRANCA SINOPOLI}

\begin{abstract}
This paper aims at defining the issue of national identity in Meneghello's oeuvre in terms of productive deformation and isotopic re-contextualization of national history. The latter is re-lived and above all re-encoded in terms of autobiographical reflexion and literary imagination, where it settles as a form of pluricultural memory. Such a memory is not referential as regards a real context which is revealed to be not recoverable. Thus the act of remembering cannot reproduce reality but produce it in its forms of "glassy essence", as the author says, of an almost immortal substance, not tied to the perishability of experience.
\end{abstract}

Con 'isotopia' ${ }^{1}$ si intende qui indicare il ripresentarsi in diversi luoghi dell'opera meneghelliana di costellazioni di riflessioni e di termini riferiti o aventi tratti semantici riferibili alla questione della 'patria' e della 'nazione', termini che vanno a materializzare uno spazio sostitutivo di quello storico-geografico reale, cioè dell'Italia. Questo spazio sostitutivo non lo è nel senso in cui duplicherebbe la realtà, rendendosi vicario di una patria e di una nazione assenti e quindi vincolandosi ad esse in un rapporto di sudditanza, di nostalgia e di debito a distanza. L'isotopia fa sì viceversa che la patria locale (cioè il paese d'origine) e la nazione possano

1 Per una definizione di "isotopia" rimando alla voce "Isotopie" di A. Saudan in Nourissier 1997:379-380. Si veda anche "Isotopia" in Segre 1985:32-34 
essere re-immaginate e prender forma e senso all'esterno delle proprie condizioni di esistenza, cioè nel divario temporale e nel distacco spaziale (in tal senso le isotopie tematiche 'patria', 'nazione', 'Italia' si rapportano all'isotopia figurale del 'dispatrio'). Com'è noto, il concetto di 'isotopia' in campo critico letterario deriva dall'uso che ne ha fatto Greimas, più di quarant'anni $\mathrm{fa}^{2}$, per indicare, tra altri aspetti, le ricorrenze semantiche, le linee guida di un testo grazie alle quali, una volta riconosciute, è possibile ottenere un effetto di coerenza del testo e di proporne di volta in volta un'interpretazione, come ha sottolineato venti anni dopo quell'intervento di Greimas un altro semiotico François Rastier. Rastier ha puntualizzato infatti, nel quadro di una semantica interpretativa, che la delimitazione di una isotopia all'interno di uno o più testi non consiste nella scoperta di qualcosa che c'è già e che va solo rilevata, ma nella congiunzione tra testo e competenza di lettura ${ }^{3}$.

L'isotopia essendo portatrice di una ridondanza semantica andrà individuata e poi correlata alla rete testuale in cui si inserisce, nella quale vivono altre isotopie, tematiche o figurative, più o meno legate a quella che ci interessa evidenziare.

Distinguo tra 'patria' e 'nazione' proprio perché, come si sa, con Meneghello siamo al cospetto di una duplicazione iniziale del problema dell'origine in quello dell'appartenenza simultanea ma non risolta al paese natale (Malo) e alla nazione (Italia). Duplicazione che grazie al "dispatrio" inglese si tramuta in una triplicazione di punti di riferimento geo-culturali e al contempo linguistici.

L'esteriorità in cui vanno a coesistere e a confliggere le tre appartenenze culturali e linguistiche è paradossalmente una interiorità, quella della memoria. La memoria cerca di avvicinare i luoghi delle diverse esperienze di appartenenza vissute dallo scrittore e di metterle in

2 Greimas 1969; vedi pure: "isotopia" in Greimas and Courtés 1979:197-199 e in Marchese 1978:133-134

3 Rastier 1987; si veda anche: Rastier 2003, in particolare 'La disontologizzazione dei testi', sul problema dell'oggettività del testo (54-7). 
una relazione produttiva, una relazione cioè in cui quei nuclei di realtà apparentemente incomunicabili possano trasfigurarsi nello spazio della scrittura che concilia materiali autobiografici e materiali letterari. A quel punto la memoria diventa una memoria scritta o meglio una scrittura di memoria (e non semplicisticamente di memorie), dove il dispositivo del ricordo funziona come meccanismo di riscrittura di una o più realtà ormai lontane e spossedute dal sé costituitosi nell'espatrio.

Per far ciò, la memoria deve tesaurizzare, interpretandola e mettendola in rilievo, proprio quella quarta dimensione che resta invisibile se si resta ancorati ai luoghi delle tre diverse appartenenze: Malo, l'Italia e l'Inghilterra. La quarta dimensione è il movimento continuo tra questi tre poli dell'identità dell'opera meneghelliana. Naturalmente essa ha avuto origine da una migrazione fisica ben concreta, tanto volontaria quanto inevitabile (vista la non corrispondenza tra $\mathrm{i}$ progetti del giovane Meneghello e l'Italia del dopoguerra) che portò nel 1947 il nostro autore a strapparsi dall'Italia e a investire il proprio progetto di vita altrove, in una nuova patria, quella inglese, immaginata attraverso le lenti rosee della cultura e quindi a sua volta duramente conquistata, anche se con un procedimento inverso che dalla astrattezza del prefigurato porta alla concretezza del vissuto. Un movimento che è esattamente l'opposto del percorso che viceversa conduce in prima battuta Meneghello ad estrarre dal vissuto irriflesso e radicato nel paese di origine quell'immaginario letterario che ricostruisce, alle condizioni della finzione letteraria, lo stesso vissuto ormai di per sé inattingibile. Non a caso la produzione di opere sul paese e sull'Italia precede quella, degli ultimi decenni, dedicata in gran parte alla riflessione sul dispatrio inglese e che si materializza nel corpus delle memorie inglesi dato da Dispatrio (1993) e dai tre volumi de Le Carte (1999-2001).

Ma nella lettura dell'opera di Meneghello, il carattere accidentale del movimento migratorio non ci porterebbe molto lontano nella ricerca di un senso che la leghi alla questione dell'identità nazionale, se non lo sostituissimo con il suo corrispettivo sul piano filosofico-letterario, cioè con il 'dispatrio'. Il dispatrio infatti non coincide con il momento del 
distacco dalla patria, ma è la traduzione/trasfigurazione letteraria del permanere in una dimensione mobile di confronto e di contatto tra poli culturali e linguistici diversi. E pertanto tale oscillazione essendo anche un 'trapianto' ha le sue fasi di rigetto, come dice Domenico Starnone nella sua lettura di Meneghello anteposta al recente volume dei Meridiani Mondadori (Meneghello 2006:XI-XLI). L'autore stesso parlava nel 2002 del 'dispatrio' nei termini seguenti:

Ho usato questa parola, 'dispatrio', è vero, ed è ciò che ti capita se oltre all'espatrio, all'uscita fisica dalla tua patria, ti senti anche cambiare dall'interno, sotto certi profili abbastanza basilari e centrali della tua vita, della tua mente e così via. Il "dispatrio" c'è stato, sono vissuto a lungo lassù.

(Mazzacurati \& Paolini 2006:29)

Al contempo, come hanno già puntualizzato altri studiosi dell'opera meneghelliana, quali Ernestina Pellegrini e Giulio Lepschy, la radice linguistica della creazione in Meneghello non avendo una funzione realistica, cioè riproduttiva della realtà, agisce da motore gnoseologico che riconfigura man mano nuovi frammenti della realtà stessa per comprenderne l'essenza, la "glassy essence", come dice Meneghello, cioè la sostanza quasi immortale che si libera dalla e della deperibilità dell'esistenza ${ }^{4}$. La ricerca e lo scavo linguistico non restano cioè confinati al piano della rappresentazione mimetica di un mondo scomparso, ma agiscono da sonde e da guida per la comprensione della realtà, atteggiamento gnoseologico sintetizzato nella frase "[...] scrivere è per me un esercizio conoscitivo" (Meneghello 2005:182).

Molto stimolante per continuare a riflettere sul senso da assegnare alla parola/dimensione di "dispatrio" è anche l'analisi di Lorenzo Chiesa, il

4 La bibliografia critica sull'opera di Meneghello è ormai molto ricca e articolata, come mostrano gli apparati bibliografici delle recenti edizioni che l'hanno ripubblicata quasi per intero. Si vedano quindi il Meridiano Mondadori Opere scelte (Meneghello 2006:1773-1801) e i precedenti volumi delle Opere (Meneghello 2007:887-899), tutti e tre a cura di F. Caputo. 
quale sostiene che il dispatrio sia leggibile come una "anamorfosi risolutiva che fornisce una chiave di lettura di cui prima non si era in possesso"; e quindi conclude la sua riflessione recuperando un significato positivo della parola stessa "dispatrio", sostenendo che:

il prefisso 'dis' non è quindi in questo caso soltanto distruttivo. [...] Oppure, in modo più radicale, dis-patrio potrebbe anche suggerire una specie di dis-integrazione, disillusione, ossimorica poiché costruttiva, riguardo a qualsiasi concetto di patria e di nazionalità. (Chiesa 2003:136)

A ben vedere, interpretare il dispatrio come 'anamorfosi' significa attribuirgli una capacità o una qualità di deformazione che consente di cogliere aspetti altrimenti trascurati o illeggibili dell'idea di 'patria/nazione'; il dispatrio, in sostanza, secondo Chiesa svelerebbe la consistenza retorica dell'identità nazionale, l'essere l'Italia un "non-luogo mentale di utopia e successiva delusione", e quindi è proprio questa artificiosità dell'identità italiana che permetterebbe di adottare uno sguardo deformante, cioè "anamorfo". Ma cos'è l'anamorfosi? È bene richiamare qui i due significati principali del termine, di derivazione pittorica e poi di adozione cinematografica. Tralasciando quelle appartenenti ad un contesto scientifico, delle sei accezioni del termine registrate dal dizionario della lingua italiana De Mauro ne restano due: la prima, di ambito pittorico, ci dice che si tratta di una "rappresentazione pittorica che esagera le distorsioni marginali proprie della prospettiva, in modo che alcune parti del soggetto siano distinguibili solo da un punto di vista diverso da quello frontale", mentre la seconda, che rimanda all'ottica, si riferisce al "fenomeno di deformazione di un'immagine sfruttato specialmente in cinematografia, per cui l'ingrandimento in senso orizzontale è diverso da quello in senso verticale" (De Mauro 2000). Da entrambe le definizioni, riportate nel contesto dell'opera di Meneghello, ricaviamo l'idea che l'Italia di cui si parla sia un'entità doppiamente artificiale: da un lato un non-luogo mentale e utopico, generato dalla 
retorica risorgimentale e poi fascista, e dall'altro una memoria interna (il cui nome è 'dispatrio') la quale funziona attraverso la creazione di ombre, tracce e calchi di eventi, oggetti e persone del passato che la scrittura letteraria fa emergere cancellandone al contempo i referenti reali.

Alle osservazioni di Lorenzo Chiesa si potrebbe aggiungere l'analisi che del termine ne ha fatto nel 2005 il critico italo-americano Peter Carravetta, il quale ritiene il dispatrio una "figura critica" che "induce a pensare a uno storcere, o sviare, o svisare la (propria) patria, o luogo/lingua nativo". Bisogna, tuttavia, a mio parere, fare attenzione al fatto che l'intervento di Carravetta sul termine meneghelliano avvenga all'interno di un discorso più generale sull'interpretazione morfosintattica e semantica della scrittura esiliata in lingua italiana, che consente di misurare la portata consistente del caso meneghelliano sul piano ben più ampio di un ripensamento critico delle coordinate stesse della letteratura degli espatriati, nel cui quadro vorrei inserire il mio discorso sull'isotopia della memoria. In sostanza Carravetta sostiene che:

La figura critica/dispatrio quindi consente di interpretare la dimensione semantica del proprio habitat (psicologico, sociale, culturale) in un senso altro da quello comunemente associato a coloro che sono costretti a trasferirsi al di fuori della patria natia o suolo nazionale, come gli espatriati, gli esiliati, i profughi o, nel termine altrove assunto come genere di cui questi sono le specie, i migranti. La nozione o parola/concetto dispatrio riporta il discorso critico/creativo 'in casa', diciamo così, e minimizza la questione dell'origine e della provenienza - che tanta parte sappiamo giocano in qualsiasi interpretazione di testi di chi per vari motivi ha dovuto 'andare via', e/o vivere in un marcato 'altrove'. Teoricamente, il dispatrio dunque è meno legato al territorio dell'espatrio [...]. (Carravetta 2005:50) 
Questa idea del 'dispatrio' come discorso di una creatività volta a ridare forma al territorio del proprio paese consente di rivedere la propria cultura o le proprie culture di appartenenza secondo prospettive nuove e secondo aspetti prima invisibili o forse inesistenti ma emersi dalla visione a distanza. Si tratterebbe, secondo Carravetta, di uno sradicamento limitato, che distinguerebbe gli scrittori del dispatrio da quelli dell'espatrio, per i quali viceversa l'esperienza della de-territorializzazione sarebbe più forte poiché condizionata da un codice (linguistico e culturale) dominante e da un contesto nuovi rispetto a quelli di origine, che non consentono di rispondere positivamente agli effetti dello spaesamento. Gli scrittori del dispatrio, invece essendo legati alla lingua e al contesto di origine (è il caso di Meneghello), riflettono sul mondo della nuova patria attraverso una comparazione continua ed ironica (non tragica) tra i due ambienti, e questo sarebbe possibile proprio grazie al fatto che si è più radicati rispetto ai primi.

In entrambi i casi, riflettendo cioè sulle due interpretazioni date da Chiesa e da Carravetta, cogliamo dunque la questione dell'identità nazionale nei termini di deformazioni, differenze, ricontestualizzazioni fittive sul piano dell'immaginario letterario in cui si sedimenta quindi una memoria non referenziale e debitrice rispetto ad un contesto di realtà, ma addirittura nei confronti di questa obliante e riscrivente ${ }^{5}$.

A questo proposito può essere utile confrontare due passi dell'opera di Meneghello, tratti dal terzo volume delle Carte, che consiste in una trascrizione di riflessioni elaborate durante la decade degli anni Ottanta. Nel primo brano, contenuto nella sezione del volume intitolata Appunti per un saggio sul dopoguerra, al cospetto del dubbio che quella particolare fase della storia d'Italia non sia più recuperabile, nemmeno attraverso il risalimento delle storie famigliari, l'io autobiografico non può che riconoscere la natura meccanica e perciò stesso 'incantatoria' della scrittura memorialistica ("È un incantagione: vorrei indurre quel tempo

5 Su tale aspetto mi permetto di rimandare, per un ampliamento, al mio contributo ““... vorrei indurre quel tempo lontano a esser stato come dico io!': la memoria e l'oblio in Luigi Meneghello" (Sinopoli 2006:37-53). 
lontano a essere stato come dico io!") (Meneghello 2001:24). Mentre nel secondo brano, tratto da un appunto datato 24 maggio 1985, descrive la natura dell'opera che sta scrivendo come fallimentare nei confronti dello stimolo da cui nasce, che è il desiderio di raggiungere la realtà, la quale però non fa che sfuggirgli:

Essenza delle CARTE, se ce n'è una: sforzo di pareggiare qualcosa, una presunta realtà effettiva di alcune cose [...]. Ogni volta che i primi segni tracciati come in trance sulla carta accennano a richiamare una realtà, comincia un intenso lavorio per raggiungerla, un fare e rifare, scavare, grattare [...]. Tutto pare artificio, tecnica da improvvisarsi piuttosto che da applicarsi, lavoro assillo. A questo punto, dov'è andata la realtà? (Meneghello 2001:286)

Sempre nello stesso volume, il terzo, delle Carte, del resto, c'è la risposta a questo scacco della conoscenza, laddove dice: "Il guaio principale è che ciò in cui si scava e colui che fa lo scavo sono la stessa persona; si può dire che la vanga con cui scavi è tra gli oggetti da scavare" (Meneghello 2001:30).

Nel quadro dunque di un generale abbandono della fiducia nella capacità referenziale del segno, che pure aveva animato la formazione filosofica di Meneghello trasformandosi, però, man mano in un progetto di comprensione della realtà in termini di scrittura letteraria, che prende forma tra l'anno dell'espatrio (1947) e quello della pubblicazione della prima opera (Libera nos a Malo, 1963), e venendo noi ora più direttamente alla congiunzione tra la memoria obliante e riscrivente e la nazione, vediamo come quest'ultima si risolva totalmente in quella grazie ad un processo di esposizione e al contempo di decomposizione degli stereotipi dell'orgoglio nazionale (anche di quelli meno altisonanti veicolati ad esempio dal cinema neorealista di De Sica), con una classica oscillazione tra serio e comico dovuta all'accostamento, costante in Meneghello, tra temi seri e messa in contesto comico dei medesimi. A 
titolo di esempio vorrei citare l'appunto o frammento del 30 settembre 1988, contenuto sempre nel terzo volume delle Carte, in cui si succedono a stretta distanza isotopie figurali e tematiche apparentemente inconciliabili quali: 'patria', 'nazione', 'deretano', 'bestia', 'urinaletto', 'toga', 'ponte di Perati', 'ladri di biciclette', 'tradizione culturale', 'squadra nazionale':

In uno dei covili (sparsi nello stanzone della testa) si riconosceva l'impronta, lo stampo deretano, del concetto di patria che ci aveva dormito. Patria, nazione: non 1'“insieme degli italiani" ma qualcosa d'altro, una bestia diversa. A volte era "ciò che sono gli italiani migliori", altre volte "ciò che sarebbero, che dovrebbero essere, che saranno [...]".

Al cinema comparivano bambini sull'urinaletto, e alcuni di noi dicevano "Questa è l'Italia vera. Sarà toga!", altri "Vergona, sputtanare la patria!"

La Patria! Inizialmente il ponte di Perati, la romanza coeva del giovanotto reduce forse proprio da Perati, che piantava i ravanelli al chiaro di luna, e a mano a mano i ladri di biciclette con la loro fatale preferenza per quelle rubate in chiave di profonda onestà.

Mio cugino si era persuaso che una patria è una tradizione culturale: Carlo Cattaneo, Benedetto Croce, Gaetano Salvemini, Piero Gobetti. Questi, con pochi altri, erano sentiti quasi come una squadra nazionale, una patria delle idee. Meno limpido il costrutto culturale dei letterati e poeti contemporanei. Mio cugino ammirava i più bravi, ma con ritegno. Gli apprezzamenti troppo generosi facevano pensare alle lodi smodate degli ungheresi (almeno quando sono in esilio, ma mi assicurano che un ungherese è sempre un ungherese, anche in patria) alla loro lingua e letteratura, e specialmente ai loro poeti. Nessuno straniero può capire quanto è straordinaria quella lingua e quanto sono bravi quei 
poeti; è una delle meraviglie della natura. (Meneghello 2001:447)

Per analizzare questo testo bisogna premettere che il primissimo dopoguerra costituisce una sorta di prova del fuoco o di cartina di tornasole per Meneghello, non solo sul piano biografico, ma anche e soprattutto a posteriori, cioè negli anni successivi del suo anglicizzarsi. Basti ricordare tre luoghi fondamentali di questa riflessione sul dopoguerra: il volume Bau-sète! (1988) e due interventi critici: il saggio Nel prisma del dopoguerra (1989, raccolto poi nel 1997 nel già citato La materia di Reading e altri reperti) e la sezione di apertura del terzo volume delle Carte, intitolata Appunti per un saggio sul dopoguerra (da cui è estrapolato il lungo brano appena citato) e genericamente attribuita ai mesi febbraio-marzo del 1980 ma che verte sugli anni dal 1945 al '47, un momento di grandi possibilità che si risolveva in un nulla di fatto, in un agitarsi invano, in un non riuscire a trovare la strada, in un non riuscire a partire con il progetto di una Italia nuova (Meneghello 2001:21), poiché la "vecchia ciurma ara ancora presente, con berretti di fortuna, sulla tolda riverniciata dell'Italia nuova![...] Ora nasceva il sospetto che rimosso il cartone e il gesso, le aquile di legno compensato, i fasci di cartapesta, le scuri friabili, stesse riemergendo più o meno intatta la sostanza di prima" (Meneghello 2001:16). Dunque un clima di grandi aspettative che si tramuta presto, per Meneghello, nella consapevolezza di non poter realizzare alcunché e quindi la decisione di espatriare.

L'appunto del 30 settembre 1988 riassume bene tale stato di delusione e di inconsistenza rispetto a quella che sarebbe dovuta essere la nuova Patria: un insieme di ipotesi ("ciò che sarebbero, che dovrebbero essere, che saranno [...][gli italiani]", sottinteso "ciò che non sono"), una successione di immagini retoriche (il ponte di Perati, canzone degli alpini della seconda guerra mondiale, sulla falsariga di quella del ponte di Bassano, gli sfortunati e onesti ladri di biciclette del film di De Sica), e infine l'astrattezza di una patria culturale fatta di filosofi antifascisti e di una ancora non ben chiara (a quei tempi) squadra di scrittori 
contemporanei più rappresentativi della nuova Italia. Efficace è comunque l'affastellarsi di tutto ciò in rapida successione paratattica, senza che si abbia il tempo di individuare l'articolarsi di una entità complessa quale dovrebbe essere la nazione.

A questo caos tuttavia premeditato o meglio consapevole ${ }^{6}$, corrisponde una fiducia nella capacità organizzativa dello sguardo della memoria letteraria, che traduce quel caos storico del dopoguerra nell'efficace nuova isotopia figurale di un 'prisma', alle cui proprietà decompositive e ricompositive bisogna affidarsi. Vorrei, in finale, richiamare l'attenzione proprio su una pagina del libro dedicato alla maturazione delle ragioni che motivarono Meneghello all'abbandono della patria, Bau-sète! (1988), quinto della serie che precede quello dedicato al Dispatrio (1993). La pagina conclude il primo capitolo, di natura introduttiva, e contiene, se così possiamo dire, tutto il "metodo" dello scrittore-filosofo ${ }^{7}$ e al contempo tutta la contraddizione da lui messa in luce tra storia e memoria letteraria:

Come spere di sole che entrino per le fessure degli scuri in un tinello buio, e vadano a colpire il prisma di cristallo posato sulla tavola, così le cose che mi sono accadute in quel tempo attraversano questo nodo prismatico in vividi fasci di raggi [...]. Per un verso le immagini ne escono con astratti pennacchi di rosso di verde di viola, come ridipinte, rinnovellate, intensificate. È un quadro più bello a vederlo, che non sia stato a viverlo [...]. Per un altro verso, tutto si deforma bruscamente: sembra che i raggi si scavezzino,

6 Sulla polifonia dei discorsi e dei generi letterari, nonché dei toni e degli stili, in particolare per quanto riguarda Libera nos a malo, è reintervenuto di recente F. Marenco nel suo "Intertestualità divertente", contributo al volume A 40 anni dal libro di Luigi Meneghello (Barbieri e Caputo 2005:45-50)

La definizione di "scrittore-filosofo" è di E. Pellegrini, che ne ha motivato le ragioni nel suo illuminante saggio La fenomenologia del Male a Malo, in Barbieri e Caputo 2005:151-163. 
spostino le cose, le vedi dove non sono, e come non sono, con improvvisi gomiti, fratture [...]. Forse questo spiega lo strano effetto a cui ho già accennato più volte, le contraddizioni dei caratteri generali che il periodo ha assunto nel mio ricordo. Credo di non dovermene preoccupare, sono tratti costitutivi, e se si contraddicono non è colpa mia: tutto ciò che posso fare è di guardare almeno nel prisma in modo relativamente ordinato, risalire intanto al principio, ai primi momenti del dopoguerra [...]. (Meneghello 1996:24)

(Università “La Sapienza”, Roma, Italy)

\section{Bibliography}

Barbieri, G. \& Caputo, F. 2005 A 40 anni dal libro di Luigi Meneghello. Vicenza: Terra Ferma.

Carravetta, $\mathrm{P}$.

Chiesa, L.

De Mauro, T.
2005 Chiasmus. Modello per l'interpretazione della scrittura esiliata o bilingue. In: Sinopoli, F. e Tatti, S. (eds.) I confini della scrittura. Il dispatrio nei testi letterari. Isernia: Cosmo Iannone.

2003 Luigi Meneghello: 'I-taglia' tra dispatrio e ‘cattivo' dialetto”. In: Burns, J. e Polezzi, L. (eds,), Borderlines. Migrazioni e identità nel Novecento. Isernia: Cosmo Iannone.

2000 Dizionario della lingua italiana per il terzo millennio. Milano: Paravia. 
Greimas, A.

Greimas, A. \& Courtés, J.

Marchese, A.

Mazzacurati, C. \& Paolini, M.

Meneghello, L.

Meneghello, L.

Meneghello, L.

Meneghello, L.

Meneghello, L.

Nourissier, F. (ed.)

Pellegrini, E.

Pellegrini, E.

Rastier, F.

Rastier, F.
1969 La semantica strutturale: ricerca di metodo. Milano: Rizzoli.

1979 Sémiotique. Dictionnaire raisonné de la théorie du langage. Paris: Hachette.

1978 Dizionario di retorica e di stilistica, arte $e$ artificio nell'uso delle parole. Milano: Mondadori.

2006 Ritratti. Luigi Meneghello. Roma: Fandango Libri.

1996 Bau-sète! Milano: Rizzoli.

2001 Le Carte. Volume III: Anni Ottanta. Milano, Rizzoli.

2005 La materia di Reading. Milano: Rizzoli.

2006 Opere scelte. Milano: Mondadori.

2007 Opere. Milano: Rizzoli.

1997 Dictionnaire des genres et notions littéraires. Paris: Albin Michel.

2002 Luigi Meneghello. Firenze, Cadmo.

2005 La fenomenologia del Male a Malo. In: Barbieri \& Caputo, 2005:151-163.

1987 Semantique interpretative. Paris: Puf.

2003 Arti e scienze del testo. Roma: Meltemi. 
Saudan, A.

Segre, C.

Sinopoli, F.
1997 Isotopie. In: Nourissier, F. (ed.), Dictionnaire des genres et notions littéraires. Paris: Albin Michel:379-380.

1985 Avviamento all'analisi del testo letterario. Torino: Einaudi.

2006 “'... vorrei indurre quel tempo lontano a esser stato come dico io!': la memoria e l'oblio in Luigi Meneghello". In: Bessière, J. (ed.), Littérature, Mémoire et Oubli/Letteratura, Memoria e Oblio. Roma: Bulzoni. 